\title{
Chapter
}

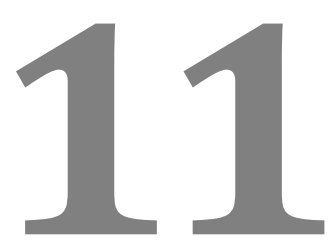

\section{A NEW PERSPECTIVE TO LIPID NANOPARTICLES FOR ORAL DRUG DELIVERY}

\author{
Neslihan Üstündağ Okur ${ }^{1 *}$, Mehmet Evren 0kur², \\ and Evren Gündoğdu ${ }^{3}$
}

${ }^{1}$ Department of Pharmaceutical Technology, School of Pharmacy, University of Istanbul Medipol, 34083 Istanbul, Turkey

2 Department of Pharmacology, School of Pharmacy, University of Anadolu, 26470, Eskisehir, Turkey

${ }^{3}$ Department of Radiopharmacy, School of Pharmacy, University of Ege, 35100 Bornova, Izmir, Turkey 


\section{Contents}

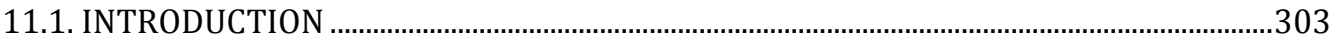

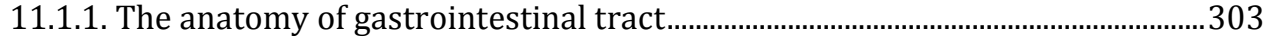

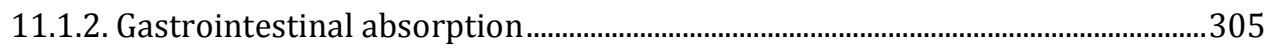

11.1.3. Transport mechanisms in the gastrointestinal tract and targeted drug

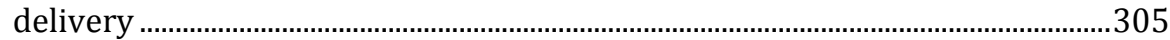

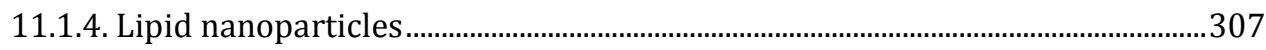

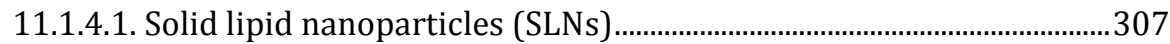

11.1.4.2. Nanostructured lipid carriers (NLCs) .....................................................307

11.1.4.3. Preparation of lipid nanoparticles .............................................................308

11.1.4.4. Toxicological effects of lipid nanoparticles.............................................308

11.1.5. Effect of characteristics of lipid nanoparticles on oral drug delivery ...............309

11.1.5.1. Particle size and release characteristics...................................................309

11.1.6. Stability of lipid nanoparticles after oral administration.....................................309

11.1.7. Strategies for oral drug delivery with lipid nanoparticles...................................310

11.1.8. Pharmacokinetic evaluations of oral lipid nanoparticles..................................... 311

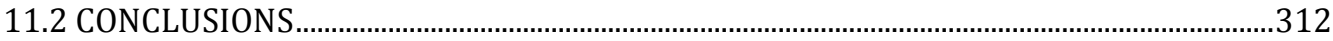

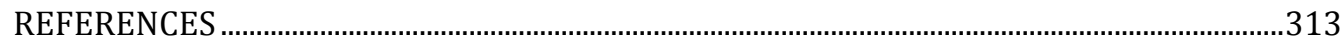




\subsection{INTRODUCTION}

Oral administration is the most commonly used and readily accepted route when compared the numerous drug delivery routes. Oral administration of drugs still remains the favored route for majority of clinical applications, due to the exceptional accessibility, and patient compliance [1]. In addition, most oral drug delivery systems such as tablets can be manufactured for comparatively and low costs because do not require sterile conditions [2-4]. Despite these advantages, the oral bioavailability of drugs is severely limited and oral formulations face several common problems, especially for peptides and proteins: (i) low stability in the gastric tract, (ii) low solubility and/or bioavailability and (iii) poor permeability across intestinal biological membranes, the mucus barrier can prevent drug penetration and subsequent absorption [5,6].

Nanoparticles, a sole subgroup of wide area of nanotechnology and they are sized between 1 and 200 nanometers. Nanoparticles may or may not display size-related properties that vary significantly from particles or bulk materials and atomic or molecular structures [7]. Lipid nanoparticles, including solid lipid nanoparticles (SLNs) and nanostructured lipid carriers (NLCs), are colloidal carriers with a lipid matrix. They are generally composed of lipids, surfactants and cosurfactant [8]. Lipid nanoparticles have been reported as an alternative drug delivery system to polymeric nanoparticles [9]. The lipid matrix is made from physiologically tolerated lipid components, which decreases the potential for acute and chronic toxicity [10].

Lipid nanoparticles are extensively employed as oral drug delivery systems, are being developed that encapsulate and protect drugs and release them in a temporally or spatially controlled manner. The nanoparticle surface can also be modified to enhance or decrease bioadhesion to target specific cells [6]. Nanoparticles are considered as alternatives to various conventional drug delivery techniques and often used to improve the oral bioavailability of drugs [7]. They can ameliorate the demerit of conventional dosage forms by prolonging the drug release, improving the drug solubility, minimizing side-effects, keeping the drug activity, and targeting [11].

\subsubsection{The anatomy of gastrointestinal tract}

To understand oral absorption of drugs the anatomy of gastrointestinal tract should be known. The anatomy of the mammalian gastrointestinal tract presents a host of impediments to the uptake of nanoparticles delivered via the oral route, including low regional $\mathrm{pH}$, a protective mucous layer, and digestive enzymes specifically designed to break down ingested proteins [12]. The digestive system contains the gastrointestinal tract and the auxiliary organs of digestion including the salivary glands, liver, gall-bladder, and pancreas. The 
gastrointestinal tract, which extracts nutrients, electrolytes, minerals, and water, is tend to injury as a result of oral drug administration [13]. The digestive system functions to ingest and digest foods, absorb necessary nutrients (proteins, carbohydrates, vitamins and minerals), and eliminate waste. The gastrointestinal tract also has a critical role in immune observation via gut-associated lymphoid tissue found throughout the tract [14]. Stomach serves the most mainly mixing part and a reservoir that secretes pepsinogen, gastric lipase, hydrochloric acid, and the intrinsic factor. This section of the gastrointestinal tract is normally impervious for absorption of most ingredients into the blood except water, ions, alcohol, and certain drugs $[15,16]$. Gastrointestinal enzymes, which contribute for presystemic metabolism of a drug, are categorized as luminal enzymes, gut wall/mucosal enzymes, bacterial enzymes. Luminal enzymes are the enzymes present in the gut fluids and contain enzymes from pancreatic and intestinal secretions. Gut wall/mucosal enzymes are primarily present in the stomach, intestine and colon [17].

Any foreign molecule that is absorbed from the gastrointestinal lumen goes across gastrointestinal mucosa, capillary beds of small and large intestine, liver via portal circulation and is then transported to the rest of the body organs. Only exception to this is the molecules absorbed into the lymphatic system or distal rectum which efficiently bypass the liver [17].

The anatomical and physiological parameters of the gastrointestinal tract strangely affect the rate and concentration of drug absorptions. There are some problems in formulating controlled release systems to be considered for better absorption and enhanced bioavailability of drugs embedded in the orally administered dosage forms. One major obligatory for the successful performance of orally administered drugs is that the drug should have good absorption throughout the gastrointestinal tract, to certify continuous absorption of the released drug [18].

A drug that is administered orally must survive transit through gastrointestinal liquids, cross the mucus layer and the epithelium before being absorbed. If most small molecules are resistant to the environment of the gastrointestinal tract and can be absorbed, the intestinal barrier limits the oral absorption of macromolecules. Hereafter, protective vehicles to avoid destruction in the gastrointestinal tract and potentially enhance oral absorption are preferred [4]. It is clear from the recent scientific literatures that an increased interest among the academic and industrial research groups still exists in formulating novel dosage forms that are retained in the stomach for a prolonged and predictable period of time [1]. For this purpose new perspective is considered for lipid nanoparticles. 


\subsubsection{Gastrointestinal absorption}

Absorption is a vital process bridging digestive system and human life. This process takes place right from the mouth to the stomach, small intestine and lastly colon [19]. Gastrointestinal tract displays site specific absorption based on the nature of drugs and regional differences such as $\mathrm{pH}$, enzyme activity, mucosa thickness, retention time and surface area $[20,21]$. The pH of gastrointestinal tract varies from 1-7, with stomach $\mathrm{pH}$ between 1-3, duodenum $\mathrm{pH}$ between 6.0-6.5, and large intestine pH from 5.5-7.0 [20,22].

In general, the gastrointestinal absorption of macromolecules and particulate materials involves either paracellular route or endocytotic pathway [23]. The paracellular route of absorption of nanoparticles employs less than $1 \%$ of mucosal surface area. Endocytotic pathway for absorption of nanoparticles is either by receptor-mediated endocytosis, which is, active targeting, or adsorptive endocytosis that does not need any ligands. This process is initiated by an unspecific physical adsorption of material to the cell surface by electrostatic forces such as hydrogen bonding or hydrophobic interactions [24].

Orally administered drugs must pass through the intestinal wall and then through the portal circulation to the liver; both are common sites of first pass metabolism (metabolism of a drug before it reaches systemic circulation). Thus, many drugs may be metabolized before adequate plasma concentrations are reached resulting in poor bioavailability [25]. Moreover permeability of drugs across the gastrointestinal membrane is one of rate limiting step in the absorption of drugs. The solubility and permeability of drugs together determine extent of oral absorption. The physicochemical factors such as $\log \mathrm{P}$, molecular weight, polar surface area, charge/ionization, number of hydrogen bond donors and acceptors determine permeability of molecule [26,27].

Liibenberg et al. were developed azithromycin nanoparticles to use perorally for AIDS therapy. The area under the curve (AUC) in the organs that are mainly infected by the human immunodeficiency virus (HIV), namely the reticuloendothelial cell containing organs, the blood, and the brain was increased [28]. In another study, SLNs of cryptotanshinone, a poorly water-soluble drug, showed twofold improved oral bioavailability than free drug due to increased solubilization capacity and change in metabolism behavior [29].

\subsubsection{Transport mechanisms in the gastrointestinal tract and targeted drug delivery}

There is one or more transport mechanisms of drugs is absorbed from gastrointestinal tract $[7,30]$ (Figure 1 ). To cross the cell membrane there are four different mechanisms: via paracellular, transcellular, carrier-mediated, and receptor-mediated transport [31]. Gastrointestinal absorption is dependent on different physical characteristics, such as molecular weight, hydrophobicity, ionization constants, and pH stability of absorbing molecules 
[31]. Due to lipid components and the surfactants containing ester groups from lipid nanoparticles is all the substrates of the hydrolysis reaction by pancreatic lipase, it is rational to deduce that lipid digestion process is inevitable in vivo, which may influence drug absorption and bioavailability [32].

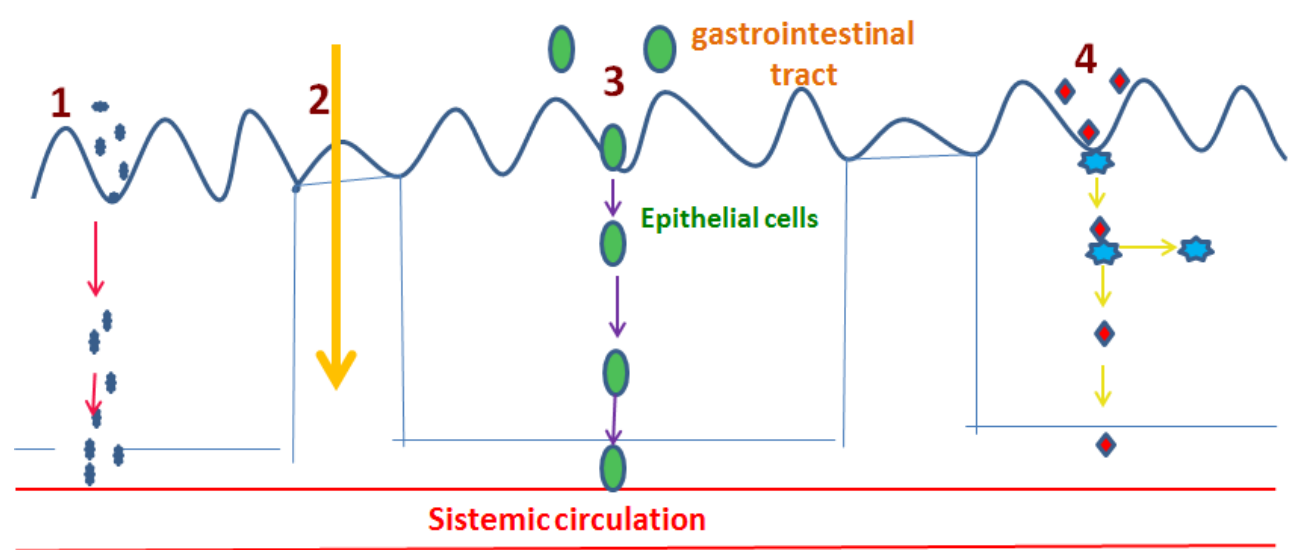

Figure 1. A schematic demonstration of absoption pathways 1) transcellular endocytosis; 2) paracellular transport and transcellular transport of drugs across the epithelial cells of the gastrointestinal tract into the systemic circulation;

3) transcellular passive diffusion; 4) carrier-mediated transport processes

To improve the oral bioavailability of nanoparticles has available many advantages. Lymphatic delivery is helpful not only for absorption of poorly soluble drugs but also for targeting drug carriers to the lymphatics. Moreover, lymphatic delivery of nanoparticles avoids the first-pass effect, and increases drug concentration in the plasma $[7,33]$.

Oral nanoparticles were found to be taken up by the gut in a lot of study. There are certain mechanisms suggested to be responsible for the peroral uptake the major site for the uptake of nanoparticles seems to be the M-cells of the Peyer's patches. Up to $60 \%$ of the uptake is accounted by these cells of the gut $[28,34]$.

Li et al. showed enhanced oral absorption of SLNs through different segments of the gastrointestinal tract. To confirm mechanistic absorption of SLNs, quarcetin-loaded SLNs were administered by oral route in rats and their pattern of absorption observed in both stomach and intestine. The results indicated that quarcetin-loaded SLNs could be absorbed in all gastrointestinal tract segments with different percentage and pattern of absorption $[7,35]$.

Hydrophilic molecules such as PEGs, carbohydrates are useful for the stabilization and diffusion of the particles in the mucus, whereas a hydrophobic coating enhances cellular and lymphatic uptake. Thiols, ammoniums and lipophilic carbon chains reinforce adhesiveness and thus 
residency time by forming disulfide bonds, electrostatic interactions and hydrophobic interactions, respectively. Moreover, the presence of acidic or functional groups leads to $\mathrm{pH}$-dependent activities that are useful for targeting different parts of the gastrointestinal tract [36].

Chitosan is a polymer that is very commonly used in oral nanoparticles. Mechanism of chitosan nanoparticles transport across gastrointestinal tract is most probably through adsorptive endocytosis. Electrostatic interaction between positively charged chitosan and negatively charged sialic acid of mucin causes association of chitosan nanoparticles to the mucus layer and then internalization via endocytosis [37-39]. Behrens et al. indicated that chitosan nanoparticles were found to be higher in the jejunum and ileum than in duodenum [40].

\subsubsection{Lipid nanoparticles}

Nanoparticles are smaller than 1 micron and possibly as small as atomic and molecular length scales $(\sim 0.2 \mathrm{~nm})$ [24]. Nanoparticles can have amorphous or crystalline form and their surfaces can act as carriers for liquid droplets or gases. They are commonly classified based on their dimensionality, morphology, composition, uniformity, and agglomeration [41]. During the last years, different materials have been entrapped into lipid nanoparticles, extending from lipophilic and hydrophilic molecules, including labile compounds, such as proteins and peptides [42-44].

\subsubsection{Solid lipid nanoparticles (SLNs)}

SLNs were developed at the beginning of the 1990s as an alternative carrier system to liposomes, emulsions and polymeric nanoparticles as a colloidal system for controlled drug delivery. SLNs consist of a solid lipid, where the drug is normally incorporated, with an average diameter below $1 \mu \mathrm{m}[7,45]$. The common excipients used in SLN formulation are solid lipids, emulsifiers, co-emulsifiers and water [46]. They display major advantages such as controlled release, improved bioavailability, protection of chemically labile molecules, cost effective excipients, enhanced drug incorporation and extensive application range [47]. Per oral administration forms of SLN may include aqueous dispersions or SLN loaded traditional dosage forms, e.g. tablets, pellets or capsules [46].

\subsubsection{Nanostructured lipid carriers (NLCS)}

NLCs are drug delivery systems composed of both solid and liquid lipids as a core matrix. NLCs disclose some advantages for drug therapy over conventional carriers, including increased solubility, the ability to enhance storage stability, improved permeability and bioavailability, reduced adverse effect, prolonged half-life, and tissue-targeted delivery [47]. 


\subsubsection{Preparation of lipid nanoparticles}

SLNs and NLCs can be produced by different formulation techniques providing reasonably high drug encapsulation efficiency. Furthermore, scaling up of most production processes can be easily succeeded [48]. SLNs are produced by replacing the liquid lipid (oil) of an oil/water emulsion by a solid or a blend of solid lipids, i.e. the lipid particle matrix being solid at both room and body temperature [49]. In the NLCs, the particles are produced using blends of solid lipids and oils. To obtain the blends for the particle matrix, solid lipids are mixed with liquid lipids. Due to the oil in these mixtures a melting point depression compared to the pure solid lipid observed, but the blends obtained are also solid at body temperature [50]. Selection of suitable lipids is essential prior to their use in preparation of lipid nanoparticles. The lipid is the main ingredient of lipid nanoparticles that influence their drug loading ability, their stability and sustained release performance of the formulations. Lipid nanoparticle dispersions based on a variety of lipid materials including fatty acids, glycerides and waxes have been investigated $[8,51]$.

\subsubsection{Toxicological effects of lipid nanoparticles}

Paralleling the development of nanoparticles, a field known as nanotoxicology has also occurred. Nanotoxicology refers to the study of the potential negative impact of the interactions between nanomaterials and biological systems $[52,53]$.

Nanoparticles can be prepared from a variety of materials such as proteins, polysaccharides and synthetic polymers. The selection of matrix materials is dependent on many factors (a) size of nanoparticles; (b) inherent properties of the drug, e.g., aqueous solubility and stability; (c) surface characteristics such as charge and permeability; (d) degree of biodegradability, biocompatibility and toxicity; (e) drug release profile desired; and (f) antigenicity of the final product [24].

A strong advantage of the use of lipid particles as drug carrier systems is the fact that the matrix is composed of physiological components, that is, excipients with generally recognized as safe (GRAS) status for oral application, which reduce the cytotoxicity $[8,42]$.

In a previous study, SLNs prepared up to concentrations of $2.5 \%$ lipid do not exhibit any cytotoxic effects in vitro [54]. Even concentrations higher than $10 \%$ of lipid have been shown a viability of $80 \%$ in culture of human granulocytes [42]. Silva et al. evaluated the toxicity of risperidone loaded SLNs with $\mathrm{Caco}-2$ cells. The results suggest that SLNs evaluated are biocompatible with $\mathrm{Caco}-2$ cells and well tolerated by the gastrointestinal tract [55]. 


\subsubsection{Effect of characteristics of lipid nanoparticles on oral drug delivery}

Nanoparticle characterization is necessary to establish understanding and control of nanoparticle synthesis and applications. It is confirmed that drug encapsulated in nanoparticles have better absorption through gastrointestinal tract as compared to their native counterpart. The factors affecting uptake include the particle size of nanoparticles and their release characteristics [56].

\subsubsection{Particle size and release characteristics}

Particle size and size distribution are the most important characteristics of nanoparticles. They affect the in vivo distribution, toxicity, drug loading, drug release and stability of nanoparticles [24]. In addition, particle size plays a key role in particle functions, such as degradation, vascular dynamics, targeting, mechanisms of clearance and uptake [57]. Particles have been shown to have different speeds, diffusion characteristics and adhesion properties, depending on their particle size [58-61].

The poor bioavailability of orally administered drugs can have mainly two reasons: low dissolution speed (i); poor permeability (ii). There is a very simple traditional approach to raise the dissolution speed by enlarging the surface, i.e. micronisation. The particle size of normally sized drug powders (in the range $20-100 \mu \mathrm{m}$ ) is reduced to a size in a range of approximately $\mathrm{l}-10 \mu \mathrm{m}$, the mean diameter being typically in the range somewhere between 2 and $5 \mu \mathrm{m}$. Nanoparticles possess sizes of approximately $10-1000 \mathrm{~nm}$; most manufacturing methods yield a main diameter somewhere between 200 and $400 \mathrm{~nm}$ [62]. Furthermore limiting nano-sized particles to less than $500 \mathrm{~nm}$ in diameter seems to be a key factor in permitting their transport through the intestinal mucosa most probably through an endocytosis mechanism [63]. In addition nanoparticles explain the size-dependent absorption mechanism based on mucoadhesion, in vivo drug release, cellular uptake, and transport across the intestinal epithelium involved in the oral absorption progression $[64,65]$.

Lipase/colipase activity is affecting the drug release from lipid nanoparticles in the gastrointestinal tract. In a pre-step of the absorption, food lipids become degraded by the lipase/colipase complex. To evaluate degradation of lipid and surfactant of the lipid nanoparticles degradation study was performed with pancreas lipase/colipase complex [42].

\subsubsection{Stability of lipid nanoparticles after oral administration}

Matrix encapsulation nature of lipid nanoparticles can protect drugs from adverse conditions encountered in the gastrointestinal tract. Metabolism of drugs especially by hydrolysis in gastrointestinal tract or in plasma can be controlled by encapsulating them in nanoparticles [19]. Nanoparticles promising an intimate contact with the intestinal mucosa are also beneficial in 
order to keep incorporated drugs towards a presystemic metabolism. Due to the close contact with the mucosa, drug degradation on the way between the delivery system and the absorption membrane is reduced to a minimum [48].

The microclimate of the stomach favors particle aggregation due to the acidity and high ionic strength. It can be expected, that food will have a large impact on nanoparticle performance [46]. For better stability of nanoparticles must be comprehensively tested due to $\mathrm{pH}$ changes and ionic strength as well as the drug release upon enzymatic degradation $[42,66]$. The idea that nanoparticles might protect labile drugs from enzymatic degradation in the gastrointestinal tract leads to the development of nanoparticles as oral drug delivery systems [67]. In addition protective effect of nanoparticles coupled with their sustained/controlled release properties prevents drugs/macromolecules from premature degradation and improves their stability in gastrointestinal tract [19].

Lipids containing a mixture of wax and glycerides may increase stability of nanoparticles than the isolated constituents due to appropriate amalgamation of different properties. Reports suggest that numerous properties of lipids such as lipid crystallization speed, recrystallization index and selfemulsification affect particle size and stability, which are major worries for the oral administration. A longer chain lipid with charge modification improves absorption and stability of nanoparticles in gastrointestinal tract [19].

\subsubsection{Strategies for oral drug delivery with lipid nanoparticles}

The lipid nanoparticles undergo digestion similarly to food lipids. Lipid nanoparticles show a high specific surface area for enzymatic attack by intestinal lipases [68]. This enzymatic degradation of the lipids leads to release of loaded drugs. The bile salts help their solubilization in the intestine and successive absorption [50]. Transport of drugs through the intestinal lymphatics via the thoracic lymph duct to the systemic circulation, avoids presystemic hepatic metabolism and then improves bioavailability of drugs [25].

Damge et al. reported that blood glucose levels were decreased at diabetic rats with oral insulin nanoparticles [69]. In another study, docetaxel loaded SLNs surface-modified with Tween 80 or d-alpha-tocopheryl polyethylene glycol succinate evaluated in terms of their feasibility as oral delivery systems. A sustained-release profile of docetaxel from the SLNs was shown. Tween 80-emulsified SLNs showed enhanced intestinal absorption, lymphatic uptake, and relative oral bioavailability of docetaxel compared with taxotere in rats. These results may be attributable to the absorption-enhancing effects of the tristearin nanoparticle. Moreover, compared with Tween 80-emulsified SLNs, the intestinal absorption and relative oral bioavailability of docetaxel in rats were further improved in d-alpha-tocopheryl polyethylene glycol succinate- 
-emulsified SLNs, It is noteworthy that these surface-modified SLNs may help as effective oral delivery systems [70].

Jain et al. developed oral insulin nanoparticles, they were able to achieve a bioavailability of up of $20 \%$ relative to injection. This enhanced bioavailability makes the delivery of insulin more possible [71].

Furthermore, when permeation enhancing polymers such as chitosans [72], carbomers [73] or thiomers [74] are used to prepare the lipid nanoparticles, the permeation enhancing property of these polymers seems to be improved. Albrecht et al. prepared diethylenetriaminepentaacetic acid gadolinium (III) dihydrogen salt loaded nanoparticles and applied orally to rats without any uptake in the systemic circulation. Due to the application of thiolated polyacrylate the condition did not change. However, when the thiolated polyacrylate was prepared with nanoparticles by the addition of poly(vinylpyrrolidone) a systemic uptake of diethylenetriaminepentaacetic acid gadolinium (III) dihydrogen salt was determined [75].

Chitosan, a cationic polymer, is widely used as a safe and effectual intestinal absorption enhancer, owing to its natural mucoadhesive property and ability to moderate the integrity of epithelial tight junctions reversibly [76]. Mucoadhesion property of chitosan can locally increase the concentration of a drug and thus raise the driving force for drug diffusion into cells, which may be useful even if the nanoparticles themselves stay trapped extracellularly in mucus [77]. In a study incubation of Caco-2 cells with chitosan-insulin loaded nanoparticles lead to in greater cell binding and uptake compared with a solution of chitosan-insulin [78]. In addition chitosan nanoparticles could also reduce the transendothelial electrical resistance (TEER) of the cell monolayers at pH 5.3 and 6.1 [79]. Moreover in vitro studies in Caco-2 cells have shown that chitosan is able to induce an opening of tight junctions thus increasing membrane permeability especially peptides and proteins [80].

\subsubsection{Pharmacokinetic evaluations of oral lipid nanoparticles}

Pharmacokinetic behaviour of drug loaded lipid nanoparticles need to distinguish if the drug is present as the released free form or as the related form with lipid nanoparticles [81]. However, it is very difficult to design of pharmaceutical formulations for the poor aqueous solubility of drugs and leads to variable bioavailability $[82,42]$.

Orally administered fenofibrate presented absorption ranging from 30 to $90 \%$ depending on gastrointestinal tract condition however; plasma level of fenofibric acid was considerably low due to rapid excretion and/or little accumulation in fat tissue. Therefore, Hanafy et al. developed fenofibrate loaded SLNs, which consequently reduced change of fenofibrate into fenofibric acid. Remarkably, fenofibrate loaded SLNs showed steady oral absorption up to $8 \mathrm{~h}$ with a stable release profile of fenofibric acid from SLNs, resulting in twofold around rise in bioavailability $[19,83]$. In another study, Cyclosporine A 
loaded SLN and Cyclosporine A solution were applied orally and were determined the plasma levels and body distribution. The incorporation into SLN protected Cyclosporine A from hydrolysis. It was found that SLN could increase bioavailability and prolonged plasma levels after per oral administration of cyclosporine containing lipid nanodispersions to animals [84]. In another study, relative bioavailability of Lopinavir from nanoparticles was 2 folds higher than the free drug [85].

Tocotrienol loaded NLCs were developed and found to be about 2-fold more effective as compared to free tocotrienol [86]. Additionally, etoposide loaded NLCs have also been applied for oral delivery and approximately 3.5-fold rise in the oral bioavailability was found as compared to free drug $[87,88]$. Zhang et al. were developed tiptolide loaded NLC and SLNs. Tiptolide loaded NLCs showed a better in vitro sustained release profile compared to tiptolide loaded SLNs. Moreover, tiptolide loaded NLCs extended mean residence time, delayed Tmax and reduced Cmax compared to free tiptolide and tiptolide loaded SLNs, respectively [89].

\subsection{CONCLUSIONS}

Effective oral drug administration is desirable but challenging owing to the nature of the gastrointestinal tract. Lipid nanoparticles are widely used to improve oral bioavailability and to achieve sustained release and to overcome hepatic first-pass metabolism effect. Oral bioavailability can be enhanced by incorporating the drugs into lipid nanoparticles possessing a solid matrix (SLN or NLC). In addition lipid nanoparticles are promising for oral and peroral administration route for drugs, proteins, and peptides. Nanoparticles can be extensively used as carriers for oral delivery, particularly for drugs having poor bioavailability. They can effectively overcome the various problems associated with oral delivery of drugs that suffers from low solubility and poor permeability, are unstable in the gastrointestinal tract and undergo extensive first pass metabolism. 


\section{REFERENCES}

1. Y. Javadzadeh, S. Hamedeyazdan. Recent Advances in Novel Drug Carrier Systems, A. D. Sezer, InTech, Rijeka, Croatia, 2012, p. 393-418.

2. A. Bernkop-Schnürch. Eur. J. Pharm. Sci. 49 (2013) 272-277.

3. S.V. Sastry, J.R. Nyshadham, J.A. Fix. Pharm. Sci. Technol. To. 3 (2000) 138-145.

4. $\quad$ L. Plapied, N.Duhem, A.Rieux, V. Préat. Curr. Opin. Colloid In. 16 (2011) 228-237.

5. M.C. Chen, F.L. Mi, Z.X. Liao, C.W. Hsiao, K. Sonaje, M.F. Chung, L.W. Hsu, H.W. Sung. Adv. Drug Deliv. Rev. 65 (2013) 865-879.

6. $\quad$ L.M. Ensign, R. Cone, J. Hanes. Adv. Drug Deliv. Rev. 64 (2012) 557-570.

7. M. Abhilash. Int. J. Pharm. Bio Sci. 1 (2010) 1-12.

8. R. Shah, D. Eldridge, E. Palombo, I. Harding. Pharmaceutical Science \& Drug Development, Springer International Publishing, New York, USA, 2015, pp. 75-97.

9. Y.F. Luo, D.W. Chen, L.X. Ren, X.L. Zhao, J. Qin. J. Control. Release 114 (2006) 53-59.

10. W. Mehnert, K. Mader. Adv. Drug Deliv. Rev. 47 (2001) 165-196.

11. S. Jana, N. Maji, A.K. Nayak, K.K. Sen, S.K.Basu. Carbohydr. Polym. 98 (2013) 870-876.

12. S.H. Bakhru, S. Furtado, A.P. Morello, E. Mathiowitz. Adv. Drug Deliv. Rev. 65 (2013) 811-821.

13. A.P. Harrison, K.H. Erlwanger, V.S. Elbrønd, N.K. Andersen, M.A. Unmack. J. Pharmacol. Toxicol. 49 (2004) 187-199.

14. K.K. Reed, R. Wickham. Semin. Oncol. Nurs. 25 (2009) 3-14.

15. G.J. Tortora, B.H. Derrickson. Principles of Anatomy and Physiology, John Wiley \& Sons, New York, USA, 2008, p. 937.

16. E.L. McConnell, H.M. Fadda, A.W. Basit. Int J Pharm 364 (2008) 213-226.

17. Y.N. Gavhane, A.V. Yadav. Saudi Pharm. J. 20 (2012) 331-344.

18. S.S. Davis. Drug Discov Today 10 (2005) 249-257.

19. H. Harde, M. Das, S. Jain. Expert Opin. Drug Deliv. 8 (2011) 1407-1424.

20. J. Renukuntlaa, A.D. Vadlapudib, A. Patel, S.H.S. Bodduc, A.K. Mitra. Int. J. Pharm. 447 (2013) 75-93.

21. U.B. Kompella, V.H. Lee. Adv. Drug Deliv. Rev. 46 (2001) 211-245.

22. K.M. Van de Graaff. Pediatr. Infect. Dis. 5 (1986) 11-16.

23. N.N. Salama, N.D. Eddington, A. Fasano. Adv. Drug Deliv. Rev. 58 (2006) 15-28.

24. V.J Mohanraj, Y. Chen. Trop. J. Pharm. Res. 5 (2006) 561-573.

25. T. Hetal, P. Bindesh, T. Sneha. Int. J. Pharm. Sci. Rev. Res. 4 (2010) 203-223.

26. C.A. Lipinski. Drug Discov. Today 1 (2004) 334-337.

27. S Basavaraj, G.V. Betager. Acta Pharmaceut. Sinic B 4 (2014) 3-17.

28. R. Liibenberg, L. Araujo, J. Kreuter. Eur. J. Pharm. Biopharm. 44 (1997) 127-132.

29. L.D. Hu, Q. Xing, J. Meng, C. Shang. AAPS PharmSciTech. 11 (2010) 1-6.

30. A.T. Florence.J Drug Target 12 (2004) 65-70.

31. Y. Yun, Y.W. Cho, K. Park. Adv. Drug Deliv. Rev. 65 (2013) 822-832.

32. Y. Liu, L. Wang, Y. Zhao, M. He, X. Zhang, M. Niu, N. Feng. Int. J. Pharm. 476 (2014) 169-177.

33. A. Bargoni, R. Cavalli, O. Caputo, A. Fundaro, M.R. Gasco, G.P. Zara. Pharm Res 15 (1998) 745-750. 
34. A.M. Hillery, P.U. Jani, A.T. Florence. J. Drug Targeting 2 (1994) 151-156.

35. H. Li, X. Zhao, Y. Ma, G. Zhai, L. Li, H. Lou. J. Control Release 133 (2009) 238-244.

36. A. Rieux, V. Pourcelle, P.D. Cani, J. Marchand-Brynaert, V. Préat. Adv. Drug Deliv. Rev. 65 (2013) 833-844.

37. T.J. Aspden, O. Skaugrud, L. Illum. Eur. J. Pharm. Sci. 4 (1996) 23-31.

38. R.J. Soane, M. Frier, A.C. Perkins, N.S. Jones, S.S. Davis, L. Illum. Int. J. Pharm. 178 (1999) 55-65.

39. M. Huang, Z. Ma, E. Khor, L.Y. Lim. Pharm. Res. 19 (2002) 1488-1494.

40. I.A. Behrens, I.V. Pena, M.J. Alonso, T. Kissel. Pharm. Res. 19 (2002) 1185-1193.

41. C. Buzea, I.I.P. Blandino, K. Robbie. Biointerphases 2 (2007) 1-103.

42. P. Severino, T. Andreani, A.S. Macedo, J.F. Fangueiro, M.H.A. Santana, A.M. Silva, E.B. Souto. J. Drug Deliv. 2012 (2012) 1-10.

43. R. Yang, R. Gao, F. Li, H. He, X. Tang. Drug Dev. Ind. Pharm. 37 (2011) 139-148.

44. M.K. Rawat, A. Jain, S. Singh. J. Pharm. Sci. 100 (2011) 2366-2378.

45. A.Z. Wilczewska, K. Niemirowicz, K.H. Markiewicz, H. Car. Pharmacol. Rep. 64 (2012) 1020-1037.

46. N. Yadav, S. Khatak, U.V.S. Sara. Int. J. Appl. Pharm. 5(2) (2013) 8-18.

47. R. Hirlekar, H. Garse, V. Kadam. Curr. Drug Ther. 6(4), 240-250.

48. A. Bernkop-Schnürch. Eur. J. Pharm. Sci. 50 (2013) 2-7.

49. R.H. Müller, K. Mader, A. Lippacher, V. Jenning. Germany Patent, DE19945203A1 (1999).

50. G.B. Singhal, R.P. Patel, B.G. Prajapati, N.A. Patel. IRJP 2(2) (2011) 40-52.

51. S. Doktorovova, R. Shegokar, L. Fernandes, P. Martins-Lopes, A. Silva, R. Müller, E.B. Souto. Pharm. Dev. Technol. 19(8) (2014) 922-929.

52. W. Jiang, B.Y.S. Kim, J.T. Rutka, W.C.W. Chan. Nat. Nanotech. 3(3) (2008) 145-150.

53. S. Bamrungsap, Z. Zhao, T. Chen, L. Wang, C. Li, T. Fu, W. Tan. Nanomedicine 7(8) (2012) 1253-1271.

54. M.A. Schubert, C.C. Müller-Goymann. Eur. J. Pharm. Biopharm. 61 (2005) 77-86.

55. A.C. Silva, E. González-Mira, M.L. García, M.A. Egea, J. Fonseca, R. Silva, D. Santos, E.B. Souto, D. Ferreira. Colloids Surf. B 86 (2011) 158-165.

56. J. Shaji, V. Patole. Indian J. Pharm. Sci. 70(3) (2008) 269-277.

57. J. Champion, Y. Katare, S.J. Mitragotri. J. Control. Release 121 (2007) 3-9.

58. V.R.S. Patil, C.J. Campbell, Y.H. Yun, S.M. Slack, D.J. Goetz. Biophys. J. 80(4) (2001) 1733-1743.

59. A.E. Nel, L. Madler, D. Velegol, T. Xia, E.M.V. Hoek, P. Somasundaran, F. Klaessig, V. Castranova, M. Thompson. Nat. Mater. 8(7) (2009) 543-557.

60. W. Zauner, N.A. Farrow, A.M.R. Haines. J. Control. Release 71 (2001) 39-51.

61. N. Yamamoto, F. Fukai, H. Ohshima, H. Terada, K. Makino. Colloids Surf. B 25 (2002) 157-162.

62. R.H. Muller, C.M. Keck. J. Biotechnol. 113 (2004) 151-170.

63. P. Jani, G.W. Halbert, J. Langridge, A.T. Florence. J. Pharm. Phamacol. 42 (1990) 821-826.

64. F. Lu, S.H. Wu, Y. Hung, C.Y. Mou. Small 5 (2009) 1408-1413.

65. C. He, L. Yin, C. Tang, C. Yin. Biomaterials 33 (2012) 8569-8578.

66. V. Mathur, Y. Satrawala, M.S. Rajput, P. Kumar, P. Shrivastava, A. Vishvkarma. Int. J. Drug Deliv. 2 (2010) 192-199. 
67. W. Tiyaboonchai. Naresuan University Journal 11 (2003) 51-66.

68. A. Bargoni, R. Cavalli, G.P. Zara, A. Fundaro, O. Caputo, M.R. Casco. Pharm. Res. 43 (2001) 497-502.

69. C. Damge, C. Michel, M. Aprahamian, P. Couvreur, J.P. Devissaguet. J. Control. Release 13 (1990) 233-239.

70. H.J. Cho, J.W. Park, I.S. Yoon, D.D. Kim. Int. J. Nanomed. 9 (2014) 495-504.

71. S. Jain, V.V. Rathi, A.K. Jain, M. Das, C. Godugu. Nanomedicine 7(9) (2012) 1311-1337.

72. M. Thanou, J.C. Verhoef, H.E. Junginger. Adv. Drug Deliv. Rev. 50 (2001) 91-101.

73. H.L. Luessen, B.J. Leeuw, M.W. Langemeÿer, A.B. Boer, J.C. Verhoef, H.E. Junginger. Pharm. Res. 13 (1996) 1668-1672.

74. K. Albrecht, A. Bernkop-Schnürch. Nanomedicine 2 (2007) 41-50.

75. K. Albrecht, M. Greindl, B. Deutel, C. Kremser, C. Wolf, H. Talasz, M.M. Stollenwerk, P. Debbage, A. Bernkop-Schnürch. J. Pharm. Sci. 99 (2008) 2008-2017.

76. T.A. Sonia, C.P. Sharma. Adv. Polym. Sci. 243 (2011) 23-54.

77. K. Bowman, K.W. Leong. Int. J. Nanomed. 1 (2006) 117-128.

78. Z. Ma, L.Y. Lim. Pharm. Res. 20 (2003) 1812-1819.

79. Z. Ma, T.M. Lim, L.Y. Lim. Int. J. Pharm. 293 (2005) 271-280.

80. V. Dodane, M.A. Khan, J.R. Merwin. Int. J. Pharm. 182 (1999) 21-32.

81. R. Li, J.S. Eun, M.K. Lee. Arch. Pharm. Res. 34 (2011) 331-337.

82. R.Mauludin, R.H. Muller, C.M. Keck. Int. J. Pharm. 370 (2009) 202-209.

83. A. Hanafy, H. Spahn-Langguth, G. Vergnault, P. Grenier, M.G. Tubic, T. Lenhardt, P. Langguth. Adv. Drug Deliv. Rev. 59 (2007) 419-426.

84. P. Ekambaram, A.H. Sathali, K. Priyanka. Scien. Rev. Chem. Commun. 2(1) (2012) 80-102.

85. P.R. Ravi, R. Vats, J. Balija, S. Prabhu, N. Adapa, N. Aditya. Carbohydr. Polym. 110 (2014) 320-328.

86. H. Ali, A.B. Shirode, P.W. Sylvester, S. Nazzal. Colloids Surf. A 353 (2010) 43-51.

87. T. Zhang, J. Chen, Y. Zhang, Q. Shen, W. Pan. Eur. J. Pharm. Sci. 43 (2011) 174-179.

88. K. Thanki, R.P. Gangwal, A.T. Sangamwar, S. Jain. J. Control. Release 170 (2013) 15-40.

89. C. Zhang, F. Peng, W. Liu, J. Wan, C. Wan, H. Xu, C. W. Lam, X. Yang. Int. J. Nanomed. 9 (2014) 1049-1063. 


\section{Chapter 11}

